Supporting information:

\title{
Diverse Transport Behaviors in \\ Cyclo[18]carbon-based Molecular Devices
}

Lishu Zhang ${ }^{\ddagger}$, Hui Lit, Yuan Ping Feng ${ }^{*}$, Lei Shen ${ }^{*}$

†Key Laboratory for Liquid-Solid Structural Evolution and Processing of

Materials, Ministry of Education, Shandong University, Jinan 250061,

People's Republic of China

‡Department of Physics, National University of Singapore, 2 Science Drive 3,

Singapore 117542, Singapore

$\S$ Department of Mechanical Engineering, National University of Singapore, 9

Engineering Drive 1, Singapore 117542, Singapore 

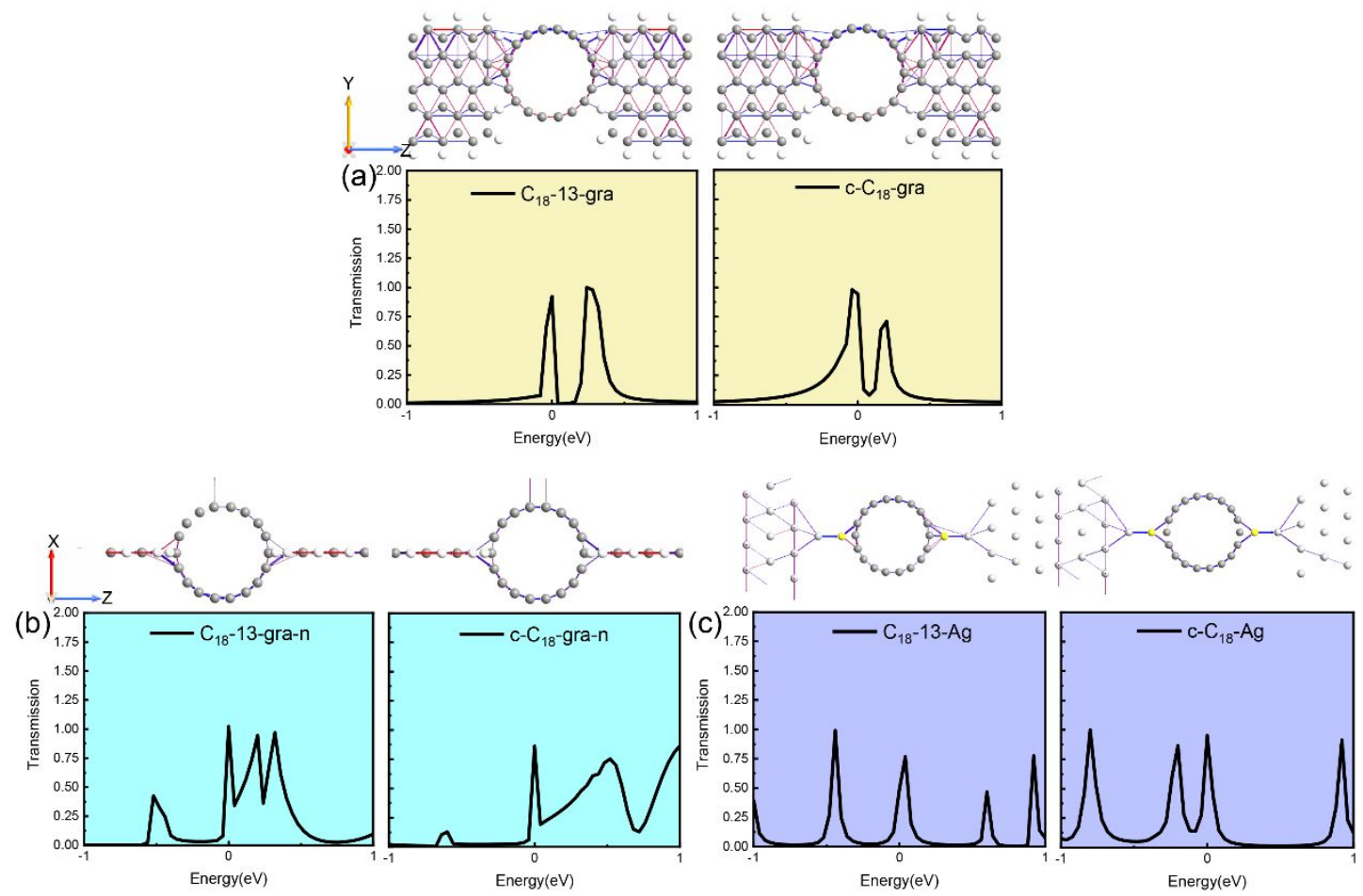

Figure S1 Equilibrium transmission pathways (top) and transmission spectra of devices of (a) p-, c- $\mathrm{C}_{18}$-gra, (b) p-, c- $\mathrm{C}_{18}$-gra-n and (c) p-, c- $\mathrm{C}_{18}-\mathrm{Ag}$, respectively. The local transmission plots are normalized with the largest arrow in each plot being the same size, irrespective of the magnitude of the total transmission at that energy. All models here are reproduced with a threshold of $5 \%$. 
(a)

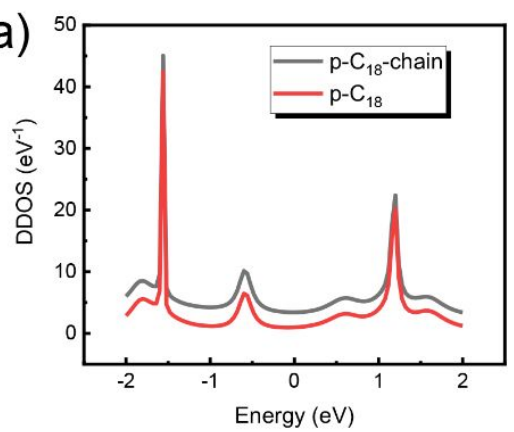

(c)

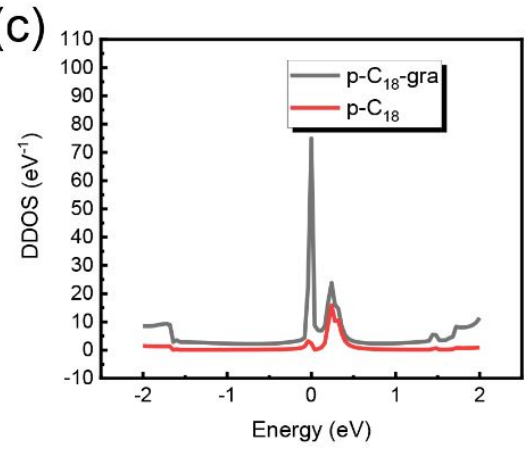

(e)

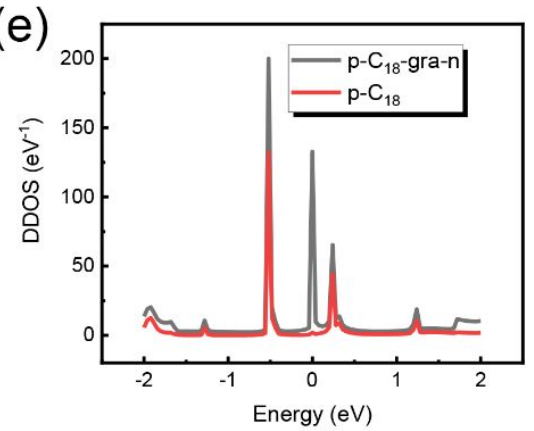

(g)

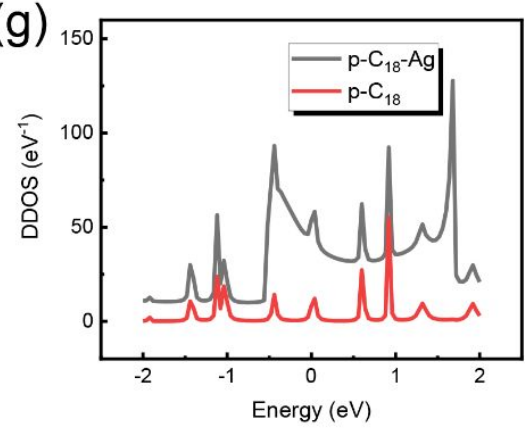

(b)

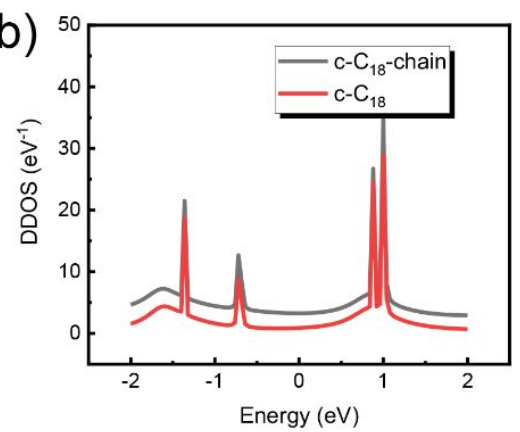

(d)

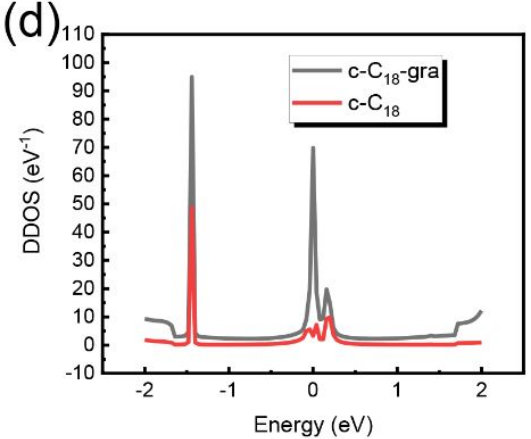

(f)

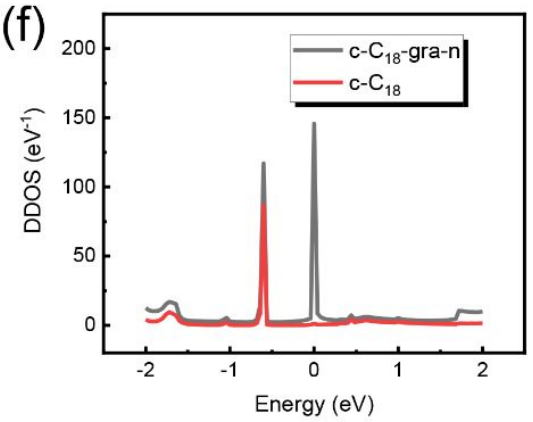

(h)

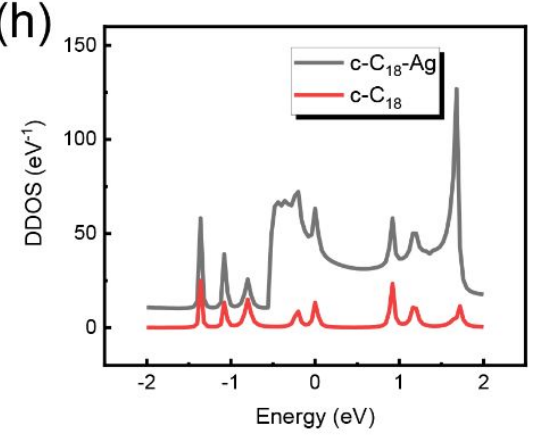

Figure S2 Device density of states of devices (a-b) p-, c-C18-gra, (c-d) p-, (e-f) c-C18gra-n and (g-h) p-, c-C18-Ag, respectively. The density of states projected on $\mathrm{C}_{18}$ are also shown by red lines. 


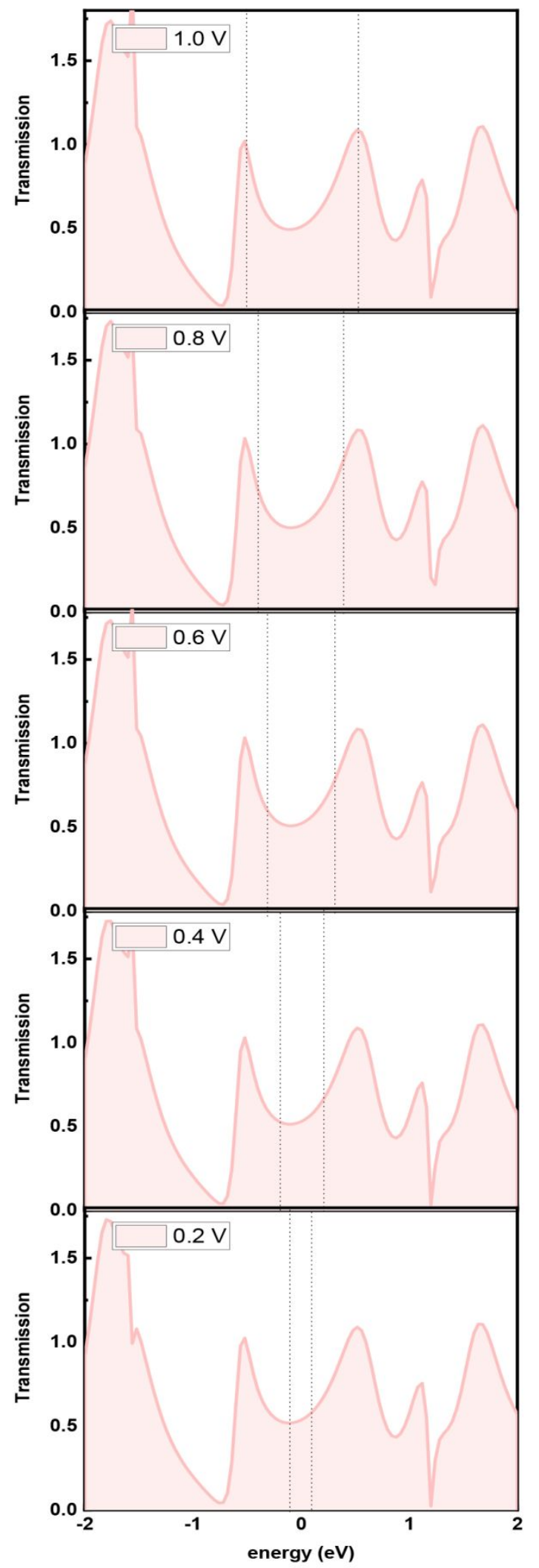

Figure S3 Transmission spectrum of $\mathrm{p}-\mathrm{C}_{18}$-chain device under different biases. The dashed line represents the bias window. 


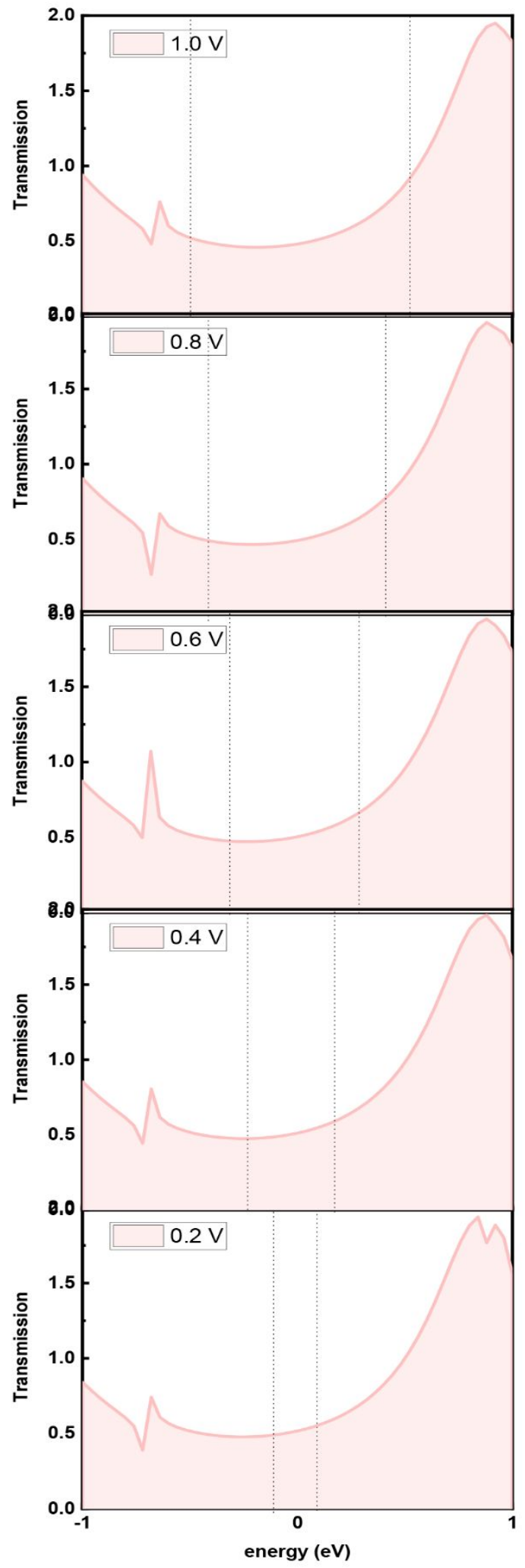

Figure S4 Transmission spectrum of $\mathrm{c}-\mathrm{C}_{18}$-chain device under different biases. The dashed line represents the bias window. 


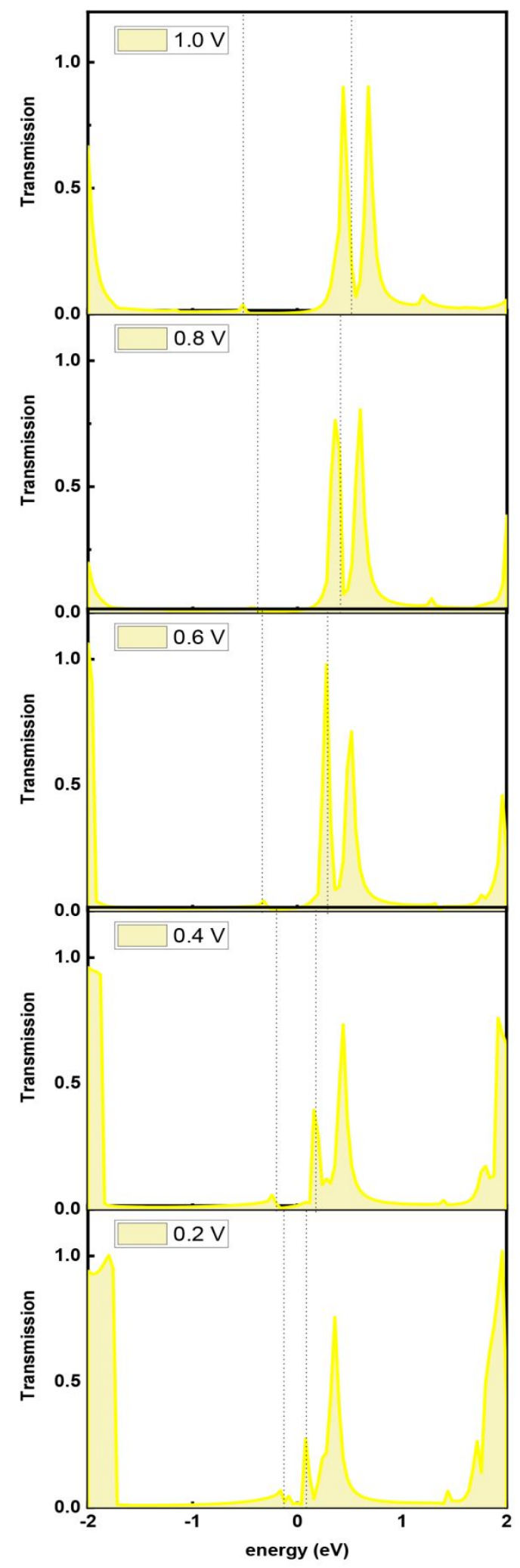

Figure S5 Transmission spectrum of $\mathrm{p}-\mathrm{C}_{18}$-gra device under different biases. The dashed line represents the bias window. 


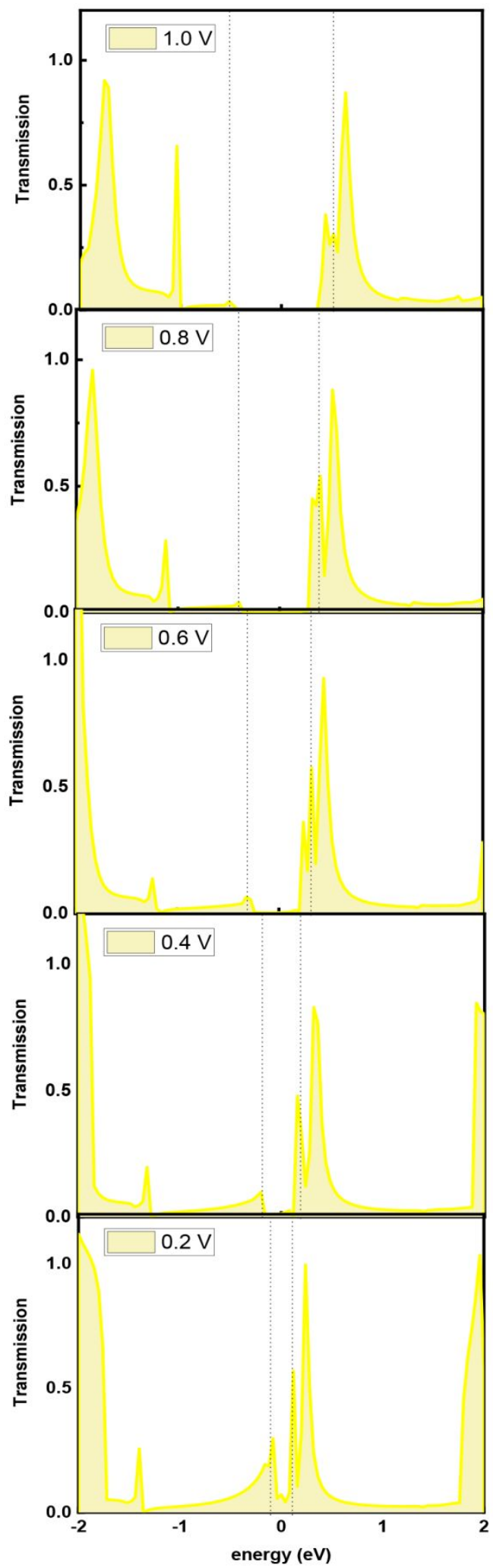

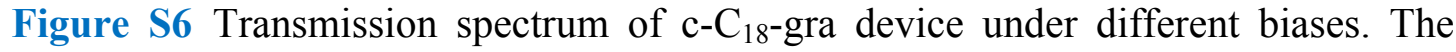
dashed line represents the bias window. 


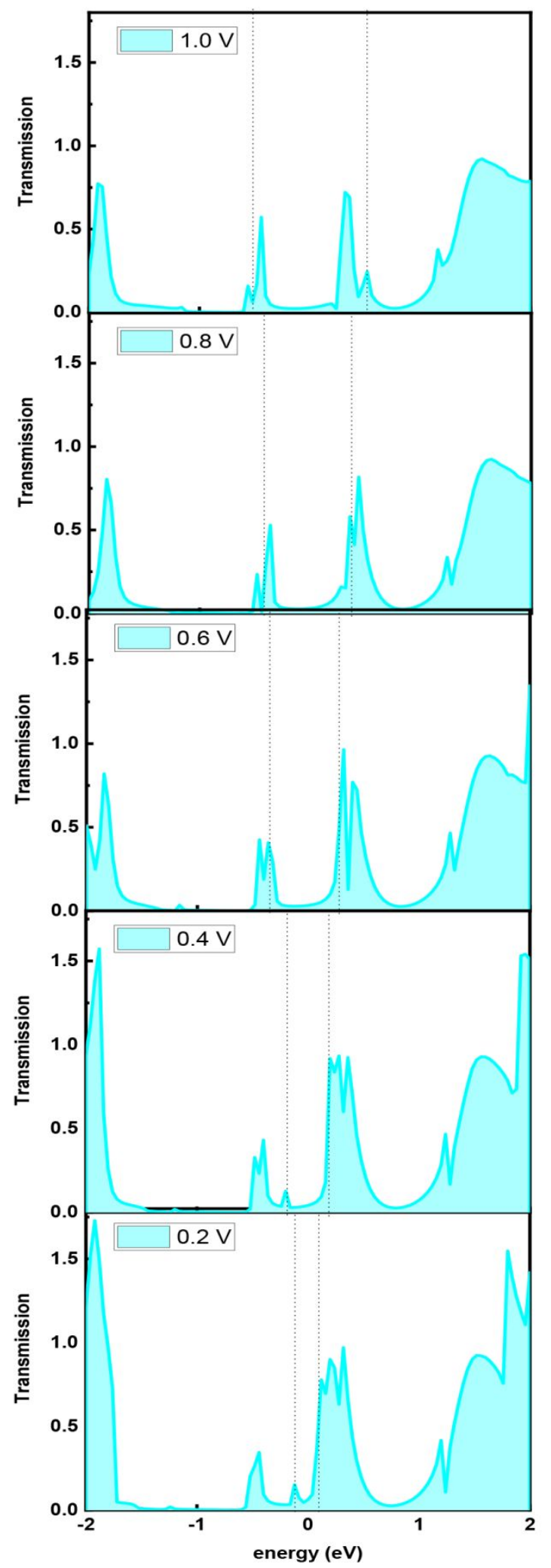

Figure S7 Transmission spectrum of $\mathrm{p}-\mathrm{C}_{18}$-gra-n device under different biases. The dashed line represents the bias window. 


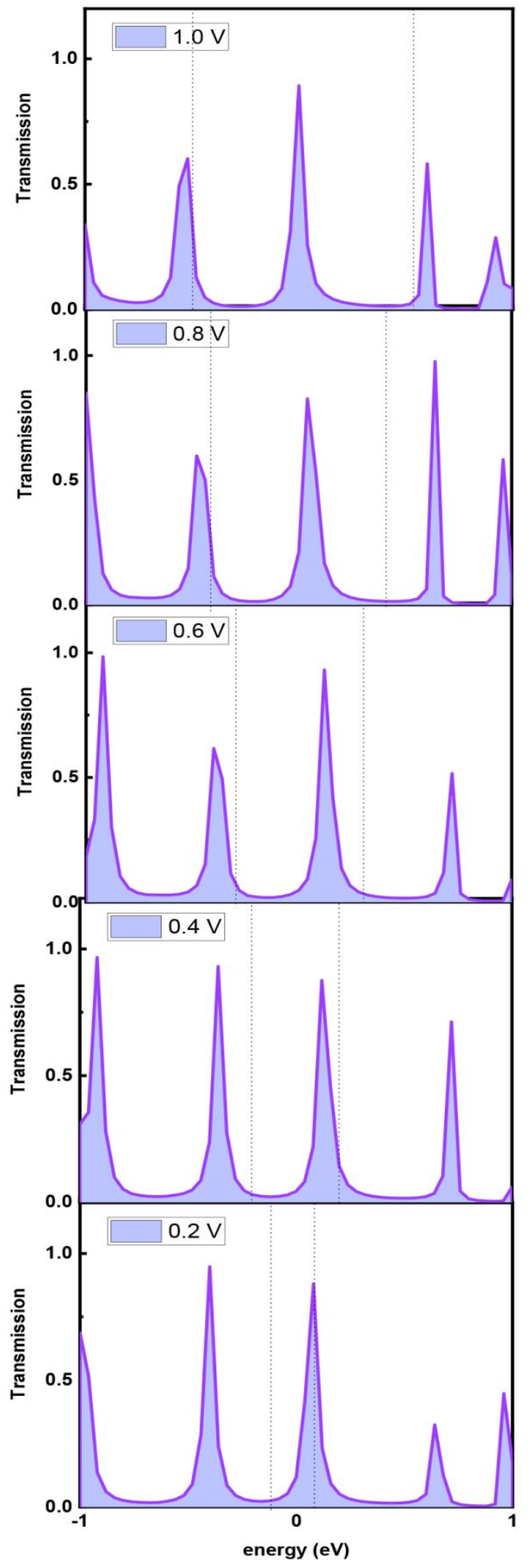

Figure S8 Transmission spectrum of $\mathrm{p}-\mathrm{C}_{18}-\mathrm{Ag}$ device under different biases. The dashed line represents the bias window. 


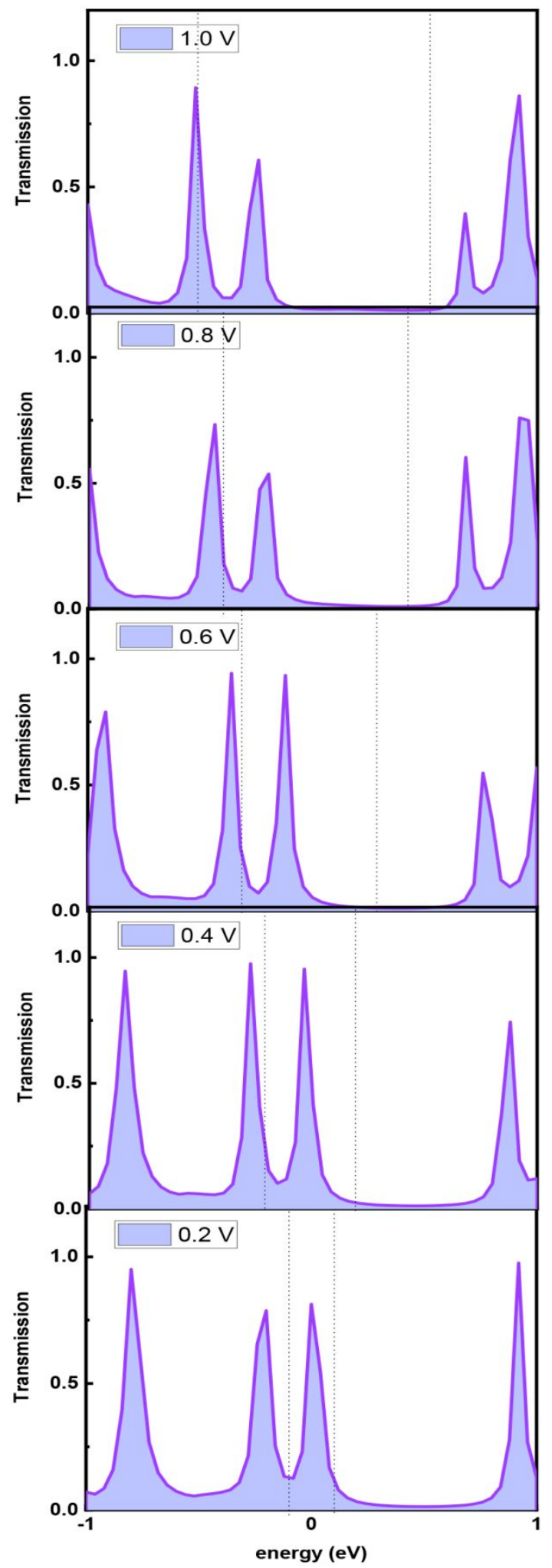

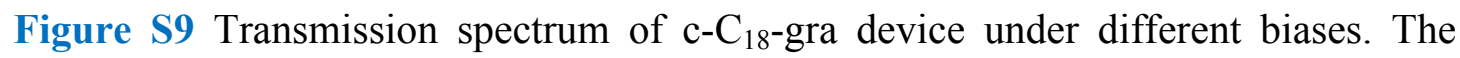
dashed line represents the bias window. 